\title{
A method of geo-acoustic parameter inversion in shallow sea by the Bayesian theory and the acoustic pressure field
}

\author{
Guangxue Zheng ${ }^{1}$, Hanhao $\mathrm{Zhu}^{1, *}$, and Jun $\mathrm{Zhu}^{2}$ \\ ${ }^{1}$ Institute of Marine Science and Technology, Zhejiang Ocean University, Zhejiang, 316022, China \\ ${ }^{2}$ Institute of Naval Architecture and Mechanical-electrical Engineering, Zhejiang Ocean University, Zhejiang, 316022, China
}

\begin{abstract}
A method of geo-acoustic parameter inversion based on the Bayesian theory is proposed for the acquisition of acoustic parameters in shallow sea with the elastic seabed. Firstly, the theoretical prediction value of the sound pressure field is calculated by the fast field method (FFM). According to the Bayesian theory, we establish the misfit function between the measured sound pressure field and the theoretical pressure field. It is under the assumption of Gaussian data errors which are in line with the likelihood function. Finally, the posterior probability density (PPD) of parameters is given as the result of inversion. Our research is conducted in the light of Metropolis sample rules. Apart from numerical simulations, a scaled model experiment has been taken in the laboratory tank. The results of numerical simulations and tank experiments show that sound pressure field calculated by the result of inversion is consistent with the measured sound pressure field. Besides, s-wave velocities, p-wave velocities and seafloor density have fewer uncertainties and are more sensitive to complex sound pressure than s-wave attenuation and p-wave attenuation. The received signals calculated by inversion results are keeping with received signals in the experiment which verify the effectiveness of this method.
\end{abstract}

\section{Introduction}

The acquisition of marine environment parameters, especially seabed acoustic parameters, plays an important role in acoustic field modeling and evaluation of underwater acoustic equipment performance[1]. Compared with the direct measurement method, the inversion by the acoustic method to obtain acoustic parameters in the test area can be more efficient. Thus, it has attracted more and more attention [2-5].

Based on the reasons above, this paper treats the complex sound pressure at each distance point received by a single hydrophone as the research object. According to Metropolis sample rule, the posterior probability distribution of three kinds of acoustic parameters, including the sound velocity, sound velocity attenuation and the density of shallow sea seabed is given. The feasibility of this method is verified by the numerical analysis and experiments in the laboratory tank experiment, which is to provide a novel idea for the inversion acquisition of geo-acoustic parameters.

\section{The inverse theory and algorithms}

\subsection{The forward model of shallow sea sound field}

Considering the influence of s-wave velocity on the

\footnotetext{
* Corresponding author: zhuhanhao@zjou.edu.cn
}

transmission of low-frequency acoustic signals in the shallow sea environment, this paper selects a shallow sea parametric model with the elastic seabed as shown in Fig.1. In the model, $z=0$ represents the sea surface, which is downward in the positive direction of the z-axis of depth. And the $r$ positive axis denotes the outward direction of sound field propagation. We suppose the depth of the uniform fluid layer to be $H$, the density to be $\rho_{1}$, and the sound velocity to be $c_{1}$. Also, the sound source is located at the depth $z_{s}$ below the sea surface. The seafloor is isotropic semi-infinite medium space. $\mathrm{S}$-wave velocities, $\mathrm{p}$-wave velocities and the seafloor density are $c_{p}, \quad c_{s}$ and $\rho_{b}, \alpha_{s}$ and $\alpha_{p}$ represent s-wave attenuation and $\mathrm{p}$-wave attenuation, which are the research object of this paper.

In Fig.1, the sound pressure $p(r, z)$ at each position in the fluid layer can be expressed as

$$
\begin{aligned}
& p(r, z)=\rho_{1} \omega^{2} \int_{0}^{\infty} \mathrm{Z}(z, \xi) \mathrm{J}_{0}(\xi r) \xi \mathrm{d} \xi \\
& \mathrm{Z}(z, r)= \begin{cases}A \cdot \sin \beta z & 0 \leq z<z_{s} \\
B \cdot \sin \beta z+C \cdot \cos \beta z & z_{s}<z<H_{1}\end{cases}
\end{aligned}
$$

where $\xi$ represents the number of horizontal waves. $r$ is horizontal distance, and $\mathrm{J}_{0}$ is the Bessel function. The derivation process and expression formulas of $A, B, C$ and $\beta$ are shown in literature[6]. There are two common methods for the numerical calculation of $p(r, z)$, which are the Normal Mode Method and the Fast Field Method (FFM). Nevertheless, when the frequency of the sound 
source is very low or the depth of sea water is extremely shallow, the fluid layer will inspire the low-order normal mode. In this case, the result of sound field obtained by the application of the Normal Mode Method will have certain errors and the FFM will be more suitable for the fast calculation of shallow sea sound field. So, in this paper, FFM is chosen for the realization the numerical calculation of pressure field $p(r, z)$ under the parameterized model as shown in Fig.1.

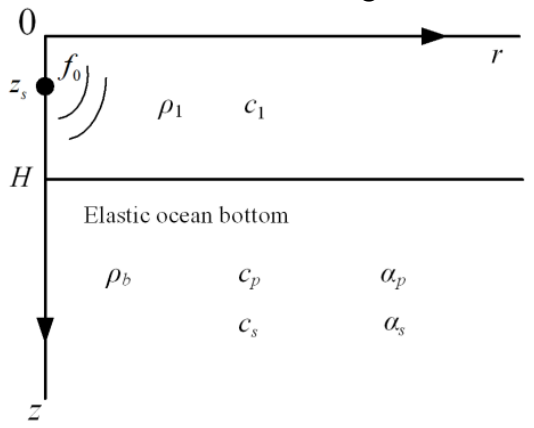

Fig. 1. A model with the elastic bottom in shallow water.

\subsection{The misfit function and the algorithm}

In accordance with the Bayesian theory, the misfit function is established based on the likelihood function under the assumption of the Gaussian data error[7,8]. The likelihood function can be considered as a function of model parameters under given measurement data, which represent the distribution of data uncertainty. $\boldsymbol{d}_{f}$ denotes the complex sound pressure (measurement field) received by $\mathrm{N}$ hydrophones, and $f=1,2,3 \ldots \mathrm{F}$ represent the observed data of $\mathrm{F}$ frequency points. Assuming that the data error is an intricate Gaussian random variable with zero mean value, the correlation between frequencies is ignored. Thus, the misfit function adopted in this paper is shown below

$$
E(\mathbf{m})=N \sum_{f=1}^{F} \log _{\mathrm{e}}\left[B_{f}(\mathbf{m})\left|\mathbf{d}_{f}\right|^{2}\right]
$$

where $B_{f}(\mathbf{m})$ represents the normalized Bartlett disqualification

$$
\mathrm{B}_{\mathrm{f}}(\mathbf{m})=1-\left|\frac{\left|\boldsymbol{p}_{f}^{+}(\mathbf{m}) \boldsymbol{d}_{f}\right|^{2}}{\left|\boldsymbol{p}_{f}(\mathbf{m})\right|^{2}\left|\boldsymbol{d}_{f}\right|^{2}}\right|
$$

where $\boldsymbol{p}_{f}(\mathbf{m})$ is the prediction of complex sound pressure (copy field) of model parameter $\mathbf{m}$. The superscript ' + ' represents conjugate transpose. In this paper, the sound pressure data of single hydrophone and single frequency point are employed in the simulation and tank experiments. Hence, equation (2) can be further simplified as

$$
\mathrm{E}(\mathbf{m})=\log _{\mathrm{e}}\left[B(\mathbf{m})|\boldsymbol{d}|^{2}\right]
$$

As can be seen from equation $(3), B_{f}(\mathbf{m})$ reaches the optimal match only when $d(r, z)=p(r, z)$. At this point, $E(\mathbf{m})$ reaches the minimum value. Through the establishment of the misfit function, the problem of geo-acoustic parameter inversion is transformed into the issue of solving the minimum value of equation (4) in the optimal range.
In this study, the simulated annealing (SA) is used to optimize five acoustic parameters in the parameter matrix $\mathbf{m}$. The posterior probability density (PPD) of parameters is given as the result of inversion according to Metropolis sample rule, and the uncertainty of inversion results is analyzed to prove the effectiveness of this method.

\section{Simulated analysis}

\subsection{Analysis of acoustic transmitting characteri- stics}

Fig.2 shows the simulation results of acoustic signals at $100 \mathrm{~Hz}$ of the sound pressure field in the range of $1-1500 \mathrm{~m}$ in typical shallow sea environment calculated by FFM, which are provided in the form of transmission loss (TL).

Table 1. The simulation parameters of ocean environment.

\begin{tabular}{|c|c|c|}
\hline Parameters & $\begin{array}{c}\text { Simulated } \\
\text { value }\end{array}$ & $\begin{array}{c}\text { Discussion } \\
\text { value }\end{array}$ \\
\hline Depth $H / \mathrm{m}$ & 100 & $/$ \\
\hline Sound velocity $c_{1} / \mathrm{m} \cdot \mathrm{s}^{-1}$ & 1500 & $/$ \\
\hline Sea-water density $\rho_{1} / \mathrm{g} \cdot \mathrm{cm}^{-3}$ & 1.025 & $/$ \\
\hline P-wave velocity $c_{p} / \mathrm{m} \cdot \mathrm{s}^{-1}$ & 2000 & 1800,2200 \\
\hline S-wave velocity $c_{s} / \mathrm{m} \cdot \mathrm{s}^{-1}$ & 1000 & 900,1100 \\
\hline Seafloor density $\rho_{b} / \mathrm{g} \cdot \mathrm{cm}^{-3}$ & 1.5 & $1.35,1.65$ \\
\hline P-wave attenuation $\alpha_{p} / \mathrm{dB} \cdot \lambda^{-1}$ & 0.5 & $0.45,0.55$ \\
\hline S-wave attenuation $\alpha_{s} / \mathrm{dB} \cdot \lambda^{-1}$ & 0.5 & $0.45,0.55$ \\
\hline
\end{tabular}

In the simulation, sound source $z_{s}$ is located at the $20 \mathrm{~m}$ underwater, with the receiving point $z$ being set at the $10 \mathrm{~m}$ underwater. And the propagation distance $r$ ranges from $1 \mathrm{~m}$ to $1500 \mathrm{~m}$. The marine environment parameters used in the simulation are shown in Table 1. In the Fig.2(A)-(E), the solid line corresponds to the calculation result of the simulated value. The point line and the dashed line correspond to the calculation result of the discussion value of five geo-acoustic parameters. In the Fig.2(F), we calculate the anomaly via regarding the TL calculated by simulated value as mean value and the TL calculated by discussion value as discrete value. Thus, the influence of the variation of each parameter on the TL can be measured by its anomaly value.

The discussion values of each parameter in table 1 are all set to the simulation value deviation $\pm 10 \%$. On this basis, the impact of the variation of each parameter on the TL is discussed.

We discover from Fig.2(A)-(E) that the change of five parameters has different influence on the TL. Under the premise that the other simulation conditions are constant, the TL varies the most when the $c_{p}$ and $c_{s}$ change. When $\rho_{b}$ alters, the change of TL is relatively prominent. When the $\alpha_{p}$ and $\alpha_{s}$ vary, the change of TL is the least obvious. Seeing from the anomaly value $s$ of five parameters in Fig.2(F), we find that, under the condition of deviation $+10 \%$, the degree of influence 
on TL in order from big to small is : $c_{p}, c_{s}, \rho_{b}, \alpha_{p}$ and $\alpha_{s}$. Under the condition of deviation $-10 \%$, the degree of influence on TL in order from big to small is: $c_{s}, c_{p}, \rho_{b}$, $\alpha_{p}$ and $\alpha_{s}$. In this regard, the influence degree of five parameters on $p(r, z)$ can be preliminarily concluded as: $c_{p}, c_{s}>\rho_{b}>\alpha_{p}$ and $\alpha_{s}$.

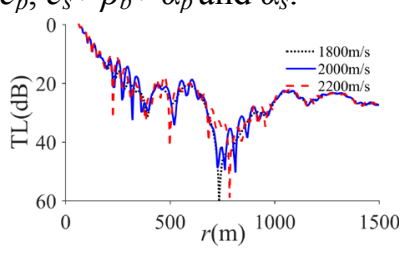

(A) $c_{p}$

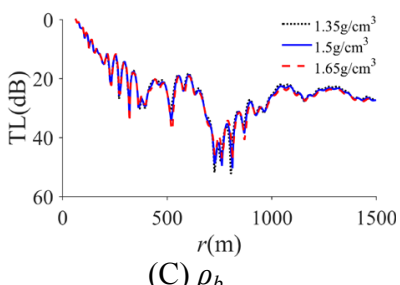

(C) $\rho_{b}$

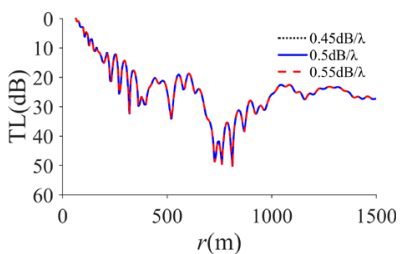

(E) $\alpha_{s}$
(B) $c_{s}$

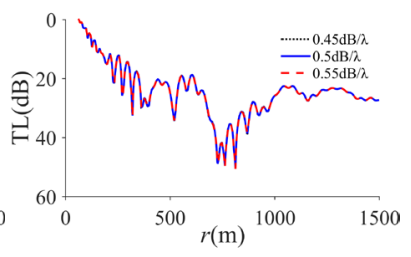

(D) $\alpha_{p}$

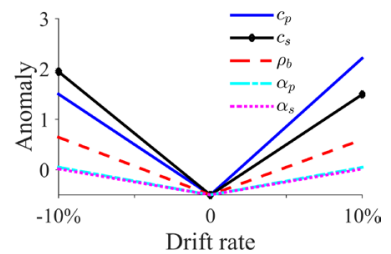

(F) Anomaly

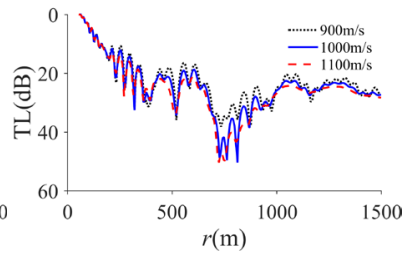

Fig. 2. The influence of five parameters on transmission loss. (A)-(E) : Changing one parameter with other parameters fixed. (F): The comparison of five parameters' anomaly.

\subsection{Sensitivity analysis of the misfit function}

Based on sound field modelling and the numerical calculation, this section discusses the sensitivity of the misfit function used in this paper to the variation of the five seabed parameters studied and analyses the reliability of the misfit function in inversion.

In the simulation, marine environment parameters are set in section 3.1, and the discussion interval of five geo-acoustic parameters is set as: (A) $c_{p}=1800 \sim 2200 \mathrm{~m} / \mathrm{s}$; (B) $c_{s}=900 \sim 1100 \mathrm{~m} / \mathrm{s} ;(\mathrm{C}) \rho_{b}=1.35 \sim 1.65 \mathrm{~g} / \mathrm{cm}^{3} ;$ (D) $\alpha_{p}=0.45$ $\sim 0.55 \mathrm{~dB} / \lambda$; (E) $\alpha_{s}=0.45 \sim 0.55 \mathrm{~dB} / \lambda . d(r, z)$ is obtained by the simulation calculation under the simulated value of environmental parameters. When discussing the sensitivity of the misfit function to a certain parameter, we adopt the fixed variable method. If other parameters remain unchanged, the parameter alters within the discussion interval to calculate the theoretical prediction value $p(r, z)$ of sound pressure field. The misfit function value $E(\mathbf{m})$ between $p(r, z)$ and $d(r, z)$ is computed by formula (4) as the sensitivity evaluation standard.

According to treatment methods above, Fig.3(A)-(E) respectively signifies the numerical changes of the misfit function $E(\mathbf{m})$ with the change of the single parameter. In each parameter discussion interval, $E(\mathbf{m})$ reaches minimum value merely at the simulated value of the parameter, which can avoid the influence of the local optimal solution on the optimization of the misfit function in the subsequent algorithm. However, with the change of those five parameters, the range of $E(\mathbf{m})$ variation is disparate. From Fig.3(F), we can see that, within the discussion interval of five parameters, the range of $\mathrm{E}(\mathrm{m})$ variation in sequence from large to small is: $c_{p}, c_{s}, \rho_{b}, \alpha_{p}$ and $\alpha_{s}$. Therefore, the influence degree of five geo-acoustic parameters on $p(r, z)$ can be defined as: $c_{p}>c_{s}>\rho_{b}>\alpha_{p}>\alpha_{s}$. It further elaborates the conclusion of section 3.1.

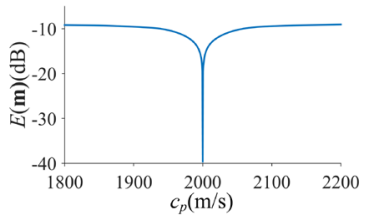

(A) $c_{p}$

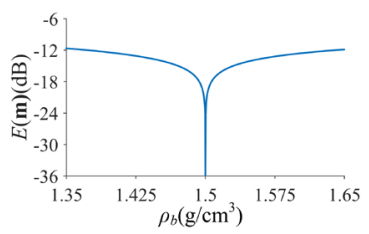

(C) $\rho b$

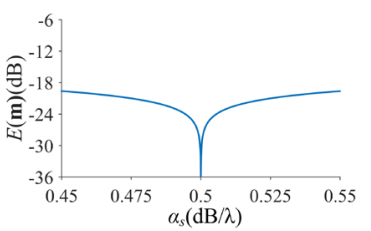

(E) $\alpha \mathrm{s}$

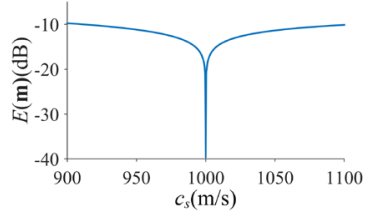

(B) $c_{s}$

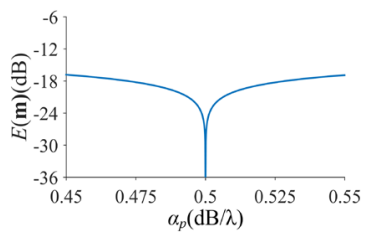

(D) $\alpha \mathrm{p}$

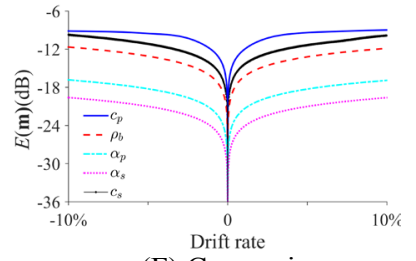

(F) Comparison
Fig. 3. The sensitivity of the single parameter. (A)-(E) :

Changing one parameter with other parameters fixed. $(\mathrm{F})$ : The comparison of five parameters' sensitivity.

\subsection{Analysis of simulation results}

Based on the discussion about sensitivity of five geo-acoustic parameters for the target function these section analyses optimization results of SA for the misfit function through the numerical simulation. Moreover, it verifies the feasibility of the inversion method studied in this paper.

In the simulation, parameters of the marine environment are same with section3.1, and the prior interval of five parameters is set in section3.2. In the SA, we set the end temperature to be $\mathrm{T}=1^{\circ} \mathrm{C}$. When the algorithm terminates, the PPD of each parameter is given in Fig.4. From Fig.4, it can be roughly seen that the peak value of the probability density of every parameter is close to its simulated value. And the sharpness of probability density curve reflects the sensitivity of the objective function to each parameter: $c_{p}, c_{s}>\rho_{b}>\alpha_{p}$ and $\alpha_{s}$. The conclusion is basically line with the conclusions in section3.1 and 3.2. Table 2 shows the setting truth value of each parameter in the simulation, the average value of SA optimization result and the variance of each parameter.

It can be discovered from Fig. 4 and Table 2 that the simulated values of each parameter are all within the mean \pm variance of their SA optimization results, which proves the feasibility of this method. 


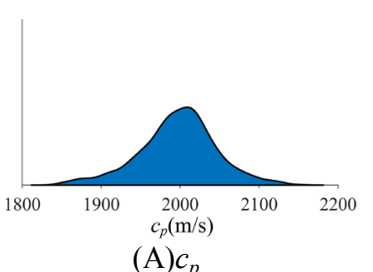

(A) $c_{p}$

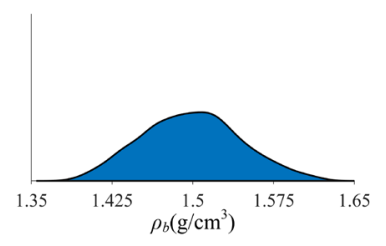

(C) $\rho b$

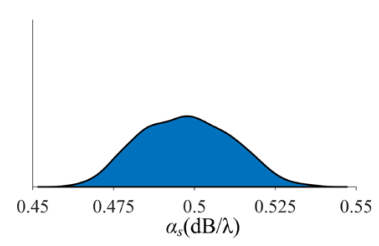

(E) $\alpha s$

Fig. 4. Five parameters and their probability density distri bution.

Table 2. The results of optimization.

\begin{tabular}{|c|c|c|}
\hline Parameters & $\begin{array}{c}\text { Simulated } \\
\text { value }\end{array}$ & $\begin{array}{c}\text { Optimization } \\
\text { result(mean } \pm \text { variance) }\end{array}$ \\
\hline$c_{p} / \mathrm{m} \cdot \mathrm{s}^{-1}$ & 2000 & $1997.2322 \pm 48.8775$ \\
\hline$c_{s} / \mathrm{m} \cdot \mathrm{s}^{-1}$ & 1000 & $1002.7605 \pm 27.8683$ \\
\hline$\rho_{b} / \mathrm{g} \cdot \mathrm{cm}^{-3}$ & 1.5 & $1.4987 \pm 0.0450$ \\
\hline$\alpha_{p} / \mathrm{dB} \cdot \lambda^{-1}$ & 0.5 & $0.4995 \pm 0.0143$ \\
\hline$\alpha_{s} / \mathrm{dB} \cdot \lambda^{-1}$ & 0.5 & $0.4975 \pm 0.0139$ \\
\hline
\end{tabular}

\section{Experimental verification}

\subsection{The tank experiment}

On the basis of the above simulation analysis, we discuss the inversion method studied in this paper by using the experimental data in this section. Our study is also based on the shrinkage ratio experiment of the anechoic tank. In the experiment, a plastic plate with high hardness and the uniform texture was selected to simulate 'semi-infinite elastic seafloor'. The transmitting transducer was fixed in one end of water, and the receiving hydrophone was fastened on the precise moving platform with grating rulers. On the grounds of frequency response characteristics of the transmitting transducer, the $\mathrm{CW}$ pulse signal with the center frequency of $135 \mathrm{kHz}$ was emitted during the experiment.

Table 3 shows measurements of parameters in the experiment. By measuring the water temperature $\left(11.15^{\circ} \mathrm{C}\right)$ through empirical formula, we can get the velocity of sound in water $c_{l}$.

During the measurement process, we keep the source fixed and transmit the $\mathrm{CW}$ signal (as shown in Fig.5) with the center frequency of $135 \mathrm{kHz}$. The hydrophone is fixed on the movable walkway, and we set the sampling rate to be $f_{s}=20 \mathrm{MHz}$. The sampling rate of each measurement point is repeated for 10 times to avoid the impact of random disturbance. After completing a measurement, the walkway drives the hydrophone to move away from the sound source by $2 \mathrm{~mm}$ (the error is no more than $20 \mu \mathrm{m})$. Fig.5(B) reflects the time domain diagram of the 50 th to 150 th receiving signal received in the measurement.

Table 3. The experiment parameters.

\begin{tabular}{|c|c|c|c|}
\hline$z_{s} / \mathrm{mm}$ & $z_{r} / \mathrm{mm}$ & $z / \mathrm{mm}$ & $c_{1} / \mathrm{m} \cdot \mathrm{s}^{-1}$ \\
\hline 87 & 84 & 182 & 1450.212 \\
\hline
\end{tabular}

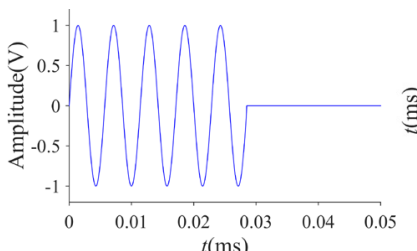

(A)

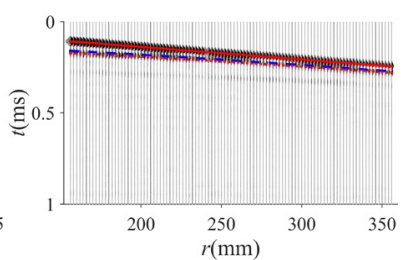

(B)
Fig. 5. (A): The signal waveform transmitted during the e xperiment. (B): The arrival time of the signal. The red se gment represents the arrival time of the direct signal; The blue dashed means the arrival time of surface reflection signal; The red dotted means the arrival time of the botto $\mathrm{m}$ reflection signal.

From Fig.5(B), we find the arrival time of each path signal obtained by the theoretical calculation to be basically consistent with the actual arrival time, which proves the reliability of the received signal in the measurement.

\subsection{Analysis of inversion results}

Table 4 lists acoustic parameters of 'seafloor', which are based on the experimental data and obtained by using the inversion method. The optimal range of five parameters is also listed as follows.

Table 4. The results of optimization

\begin{tabular}{|c|c|c|}
\hline Parameters & Optimal range & $\begin{array}{c}\text { Optimization } \\
\text { result(mean } \pm \\
\text { variance) }\end{array}$ \\
\hline$c_{p} / \mathrm{m} \cdot \mathrm{s}^{-1}$ & $2200-2500$ & $2399.3637 \pm 69.6299$ \\
\hline$c_{s} / \mathrm{m} \cdot \mathrm{s}^{-1}$ & $1100-1300$ & $1242.9780 \pm 41.8826$ \\
\hline$\rho_{b} / \mathrm{g} \cdot \mathrm{cm}^{-3}$ & $1.0-1.8$ & $1.2109 \pm 0.1777$ \\
\hline$\alpha_{p} / \mathrm{dB} \cdot \lambda^{-1}$ & $0.1-1.1$ & $1.0298 \pm 0.2122$ \\
\hline$\alpha_{s} / \mathrm{dB} \cdot \lambda^{-1}$ & $0.1-1.1$ & $0.1876 \pm 0.1697$ \\
\hline
\end{tabular}

In the processing of experimental data, the SA Settings are identical to the ones in section 3.3. Fig.6 shows the PPD of each parameter when the algorithm terminates.

The magnitude of the variance represents the magnitude of the uncertainty. From Table 4 and Fig. 6 we can draw a conclusion that: $c_{p}, c_{s}$ and $\rho_{b}$ have fewer 
uncertainties and are more sensitive to complex sound pressure than $\alpha_{p}$ and $\alpha_{s}$. It's worth mentioning that the density of the plastic plates is known in the experiment, which is $1.2 \mathrm{~g} \cdot \mathrm{cm}^{-3}$. It is very close to the inversion result, which verifies the reliability of the experimental results.

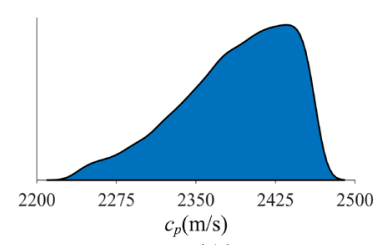

(A) $c_{p}$

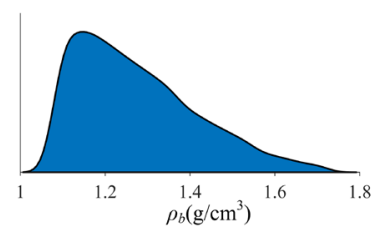

(C) $\mathrm{pb}$

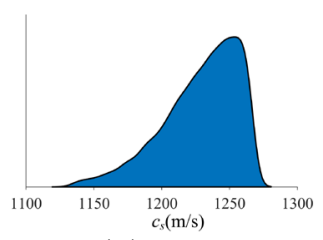

(B) $c_{s}$

(D) $\alpha \mathrm{p}$
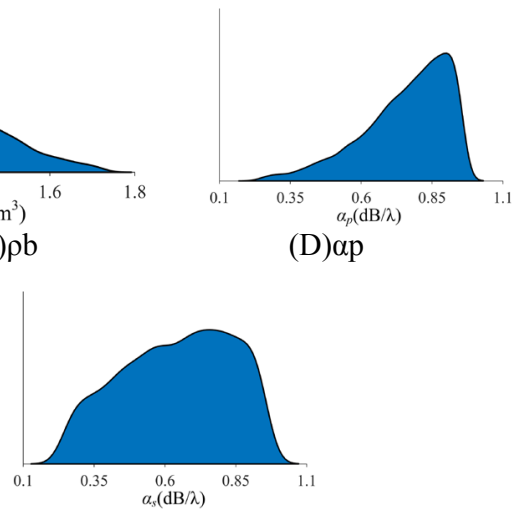

(E) $\alpha \mathrm{s}$

Fig. 6. Five parameters and their probability density distri bution.

\subsection{The verification}

In order to verify the effectiveness of the proposed method, the received signal is compared with the calculated signal in this section.

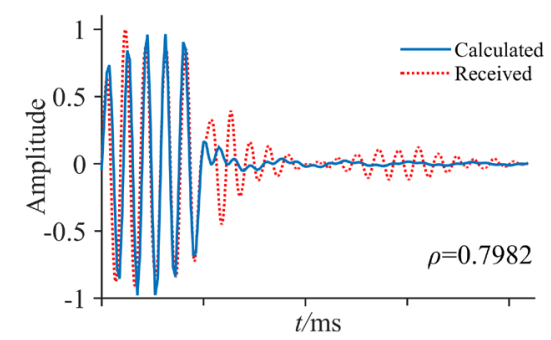

Fig.7. The comparison of two kinds of signal. The red dot line represents the received signal and the blue line means the calculated signal by the result of inversion. ' $r$ ' is correlation coefficient.

The received signal at $1 \mathrm{~m}$ is calculated by result of inversion (mean of each parameter). Then find the corresponding receiving point and extract the signal. Fig. 6 shows a very high correlation between those two kinds of signal. It proves that the inversion result is credible.

\section{Summary}

In this paper, a method of geo-acoustic parameter inversion in shallow sea based on Bayesian theory is studied. The result of research shows that under the misfit function given in this paper, applying the Bayesian inversion theory, the PPD of the parameters can be obtained. Simulation and experimental results verify the effectiveness of the proposed method.

This work was supported by the National Natural Science Foundation of China (11704337), State Key Laboratory of Acoustics, Chinese Academy of Sciences (SKLA201901) and Key Laboratory of Ocean Observation-Imaging Tested of Zhejiang Province (00IT20180F05).

\section{References}

1. P. Gerstoft. J. Acoust. Soc. Am. 95, 70-782 (1994).

2. Hanhao Z, Shengchun P, Haigang Z. J. Harbin Engineering Univ. 33 (2012).

3. C H. Harrison, P L. Nielsen. J. Acoust. Soc. Am., 121(1): 108-119 (2007).

4. S E. Dosso, C W. Holland. IEEE J. Ocean. Eng. 31(3): 657-671(2006).

5. A L S. Lapinski, S E. Dosso. IEEE J. Ocean. Eng. 28(3): 380-393 (2003).

6. Hanhao Z, Harbin Engineering Univ. (2014).

7. S E. Dosso, P L. Nielsen, M J. Wilmut. J. Acoust. Soc. Am. 119(1): 208-219 (2006).

C F. Huang, P. Gerstoft, W S. Hodgkiss. J. Acoust. Soc. Am. 119(1): 197-207 (200 